\title{
'Diseases Desperate Grown by Desperate Appliance Are Relieved, or Not at all' - Towards Finding a Cure for Pancreatic Cancer, Where Do We Stand Today?
}

\author{
Georg Feldmann ${ }^{a}$ Peter Brossart ${ }^{a} \quad$ Anirban Maitra $^{b}$ \\ a Department of Internal Medicine 3, University Hospital of Bonn, Bonn, Germany; \\ b Sheikh Khalifa Bin Zayed Al Nahyan Distinguished University Chair in Cancer Research, University of Texas, \\ MD Anderson Cancer Center, Houston, TX, USA
}

Recent decades have seen vast improvements in the management of several of the worst human maladies including various forms of malignant neoplasias. However, despite considerable efforts both in the clinic as well as at the laboratory bench and despite introduction of innovative novel therapy regimens, prognosis and overall survival of patients suffering from pancreatic ductal adenocarcinoma (a.k.a. pancreatic cancer) have only marginally improved since the days the legendary Prince of Denmarke's story was written. Pancreatic cancer still is a 'disease desperate grown' and there are projections estimating that pancreatic cancer might soon become the second most common cause of cancer-related death in Western countries [1].

It remains an important goal to enhance quality of life and longterm survival, and ultimately to find a cure for all patients affected by this diagnosis. However, an ever-growing body of evidence suggests that these aims might be difficult to fully achieve in the near future. There are, nevertheless, certain vulnerabilities of this entity that we might be able to exploit for a direct, clinically relevant impact for defined subsets of pancreatic cancer patients. Exploiting these vulnerabilities and translating them into safe, standardized clinical procedures will require adamant, internationally concerted research efforts by both basic and clinical researchers. These efforts need to be aided by public awareness and sufficient financial support.

The mini-review series in the current issue of ONCOLOGY Research and Treatment aims to contribute to this effort by providing a concise overview of state-of-the-art clinical care, currently ongoing developments in pancreatic cancer translational research as well as by pinpointing some potential future directions. The introductory article by Bisht et al. [2] gives a summary of the current standard of care for advanced pancreatic cancer and sums up the most relevant ongoing clinical trials.
Development and routine clinical implementation of strategies for early detection of pancreatic cancer likely carry enormous potential to improve the overall outcome for virtually all cases of pancreatic cancer [3]. The article by Kim and Hong [4] gives an up-todate review of our current understanding of precursor lesions to pancreatic cancer and underlying molecular alterations, thus describing the molecular basis of finding potential targets for tests to detect high-risk precursor lesions prone to progress into fully invasive and subsequently metastatic pancreatic cancer. Another highly relevant topic for development and implementation of clinical procedures for early detection is the field of hereditary pancreatic cancer. This topic, which is covered virtuously by a brilliant piece of work by Benzel and Fendrich [5], is also important for a better understanding of pancreatic carcinogenesis and secondary prophylaxis. Together, both authors oversee a considerable fraction of all cases of familial pancreatic cancer recorded in Germany.

A brief overview of currently hot topics in translational research on pancreatic cancer, e.g. identification of actionable mutations and development of novel molecularly targeted therapeutic approaches is provided by Bisht and Feldmann [6]. Defective response to DNA damage has been identified as one of the most promising targets in gastrointestinal cancers, including pancreatic cancer. These targets are likely particularly prone to respond exceptionally well to targeted therapeutic intervention, including radiotherapeutic and immunological approaches as well as molecularly targeted compounds and certain classical cytostatic agents. This extremely interesting developing field is made accessible by the skilled overview provided by Schmitt et al. [7].

It is our sincere hope that this collection of focused reviews will make its contribution to foster public awareness and support for pancreatic cancer translational research and help in giving interested clinicians and basic researchers alike an up-to-date overview of ongoing efforts.

\section{KARGER}

() 2018 S. Karger GmbH, Freiburg 


\section{Disclosure Statement}

The authors declare no conflict of interests that might impair the impartiality.

\section{References}

1 Rahib L, Smith BD, Aizenberg R, Rosenzweig AB, Fleshman JM, Matrisian LM: Projecting cancer incidence and deaths to 2030: The unexpected burden of thyroid, liver, and pancreas cancers in the United States. Cancer Res 2014;74:2913-2921.

2 Bisht S, Feldmann G, Brossart P: Current therapeutic options for pancreatic ductal adenocarcinoma (PDAC) Oncol Res Treat 2018; 41 DOI: 10.1159/000493868.
3 Yachida S, Jones S, Bozic I, Antal T, Leary R, Fu B, Kamiyama M, Hruban RH, Eshleman JR, Nowak MA, Velculescu VE, Kinzler KW, Vogelstein B, IacobuzioDonahue CA: Distant metastasis occurs late during the genetic evolution of pancreatic cancer. Nature 2010; 467:1114-1117.

4 Kim JY, Hong SM: Precursor lesions of pancreatic cancer. Oncol Res Treat 2018; 41 DOI: 10.1159/000493554.
5 Benzel J, Fendrich V: Familial pancreatic cancer. Oncol Res Treat 2018; 41 DOI: 10.1159/000493473.

6 Bisht S, Feldmann G: Novel targets in pancreatic cancer therapy. Oncol Res Treat 2018; 41 DOI: 10.1159 / 000493437.

7 Schmitt A, Reinhardt C, Zander T, Feldmann G: Targeting defects in the cellular dna damage response for the treatment of pancreatic ductal adenocarcinoma. Oncol Res Treat 2018; 41 DOI: 10.1159/000493401. 